\title{
On the use of non-linear vibrations and the anti-resonances of Higher-Order Frequency Response Functions for crack detection in pipeline beam
}

\author{
Jean-Jacques Sinou \\ Laboratoire de Tribologie et Dynamique des Systèmes UMR-CNRS 5513 \\ Ecole Centrale de Lyon, 36 avenue Guy de Collongue \\ 69134 Ecully Cedex, France \\ jean-jacques.sinou@ec-lyon.fr
}

\begin{abstract}
The identification of new scientific challenges, as well as the increasing high-performance computing support, indicate that the benefits of applying novel nonlinear techniques for crack detection will continue to grow. So, significant effort has been invested in recent years to develop effective techniques to detect crack in mechanical structures. The objective of this paper is to discuss and propose a robust diagnostic of damage based on non-linear vibrational measurements with particular regard to the Higher-Order Frequency Response Functions. An important observation is that the appearances of the non-linear harmonic components and the emerging anti-resonances in Higher-Order Frequency Response Functions can provide useful information on the presence of cracks and may be used on an on-line crack monitoring system for small levels of damage. Efficiency of the proposed methodology is illustrated through numerical examples for a pipeline beam including a breathing crack.
\end{abstract}

\section{Introduction}

The presence of a crack may lead to serious damage and dangerous effect on the dynamic behavior of mechanical structures. In order to avoid severe damage, the detection of a crack in the early stage can be of great interest. In recent years a lot of effort has been devoted to the detection of cracks in mechanical structures and many researchers have proposed various developments of non-destructive techniques based on changes in the structural vibrations [1-4]. Basic methods based on linear condition monitoring techniques have been extensively developed by considering not only changes in natural frequencies and modes shapes [5], but also the appearances of resonant peaks due to vibration coupling [6,7], different changes in the measurements of the Frequency Responses Functions and the motion of anti-resonances [8-10].

Even if in the case of simple structures, the damage severity can be determined from changes by using various linear analysis previously presented (changes in natural frequencies, modes shapes, Frequency Response Functions, anti-resonances, coupling measurements,...), some researchers have illustrated the fact that the presence of damage can induce more complicated behavior. For example Gudmundson [11] noted that decrease in natural frequencies for a cantilever beam is not always observed during experimental tests due to the closing of the crack. So the linear analysis appears to be insufficient to describe the non-linear behavior of the crack, the so called "breathing phenomena" corresponding to the fact that the crack alternately opens and closes during experimental tests. Also, the common use of robust developments based on the presence of the nonlinear components in the vibrational responses of damaged structures has to be taken into account to avoid worse damage identification $[4,12,13]$.

However few efforts have been dedicated to discuss the main advantages of using the non-linear vibrational signatures and anti-resonances of the Higher-Order Frequency Response Functions for an efficient and robust 


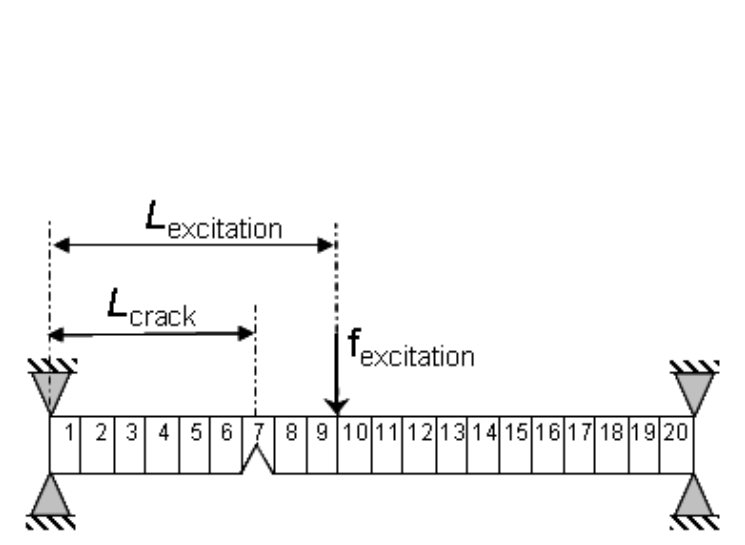

(a) Description of the pipeline beam system

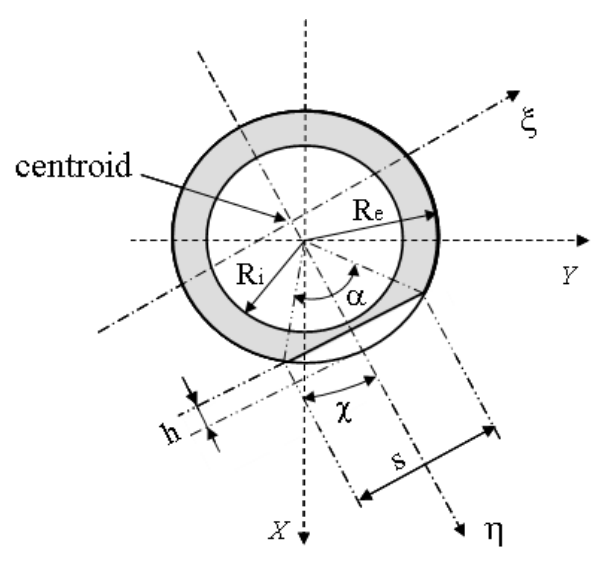

(b) Crack element

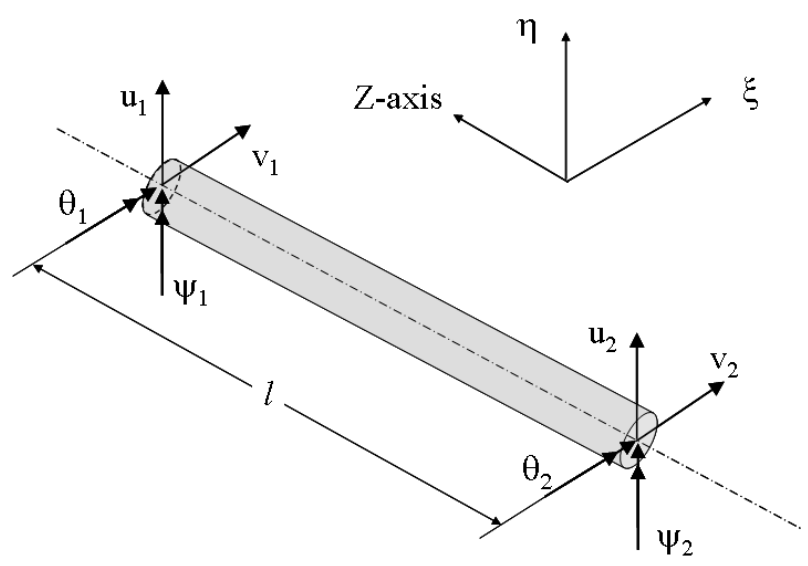

(c) Description of the degrees of freedom in $\eta$ and $\xi$-axes

Figure 1: Finite-element model of the pipeline beam and the crack section

detection and localization of damage. In order to propose some answers, the present study proposes to show that not only an appropriate use of the super-harmonic frequency components of the non-linear dynamical behaviour but also the anti-resonances of the super-harmonics components can be useful for crack identification in mechanical systems. The paper is organized as follows: firstly, a description of the cracked simply supported pipeline beam and the estimation of the non-linear vibrational response of the mechanical system are discussed. Secondly, explanation is given of the most common uses of the non-linear signatures to detect the presence of damage. Finally, damage detection and detection of the crack location based on the emergence of antiresonances for the super-harmonics components are discussed.

\section{The model of the cracked beam}

The system is composed of a beam of length $0.5 \mathrm{~m}$ with an inner and outer diameters of $0.06 \mathrm{~m}$ and $0.1 \mathrm{~m}$ respectively (see Figure 1(a)). The beam is discretized into 20 Timoshenko beam finite elements, with four degrees of freedoms at each node, the axial and torsional degrees of freedom being not considered. The pipeline beam is simply supported at each end, as indicated in Figure 1(a)). The layout of the cracked beam element under consideration is shown in Figure 1(b). All the values of the material properties and dimensions of the beam are given in Table 1. 
The equations of the simply supported cracked pipeline beam can be written as

$$
\mathbf{M} \ddot{\mathbf{x}}+\mathbf{C} \dot{\mathbf{x}}+(\mathbf{K}+\mathbf{q}(t)) \mathbf{x}=\mathbf{f}
$$

where $\ddot{\mathbf{x}}, \dot{\mathbf{x}}$ and $\mathbf{x}$ are the acceleration, velocity and displacement vectors. $\mathbf{M}, \mathbf{C}$ and $\mathbf{K}$ are the mass, damping and stiffness matrices of the complete uncracked pipeline beam. $\mathbf{f}$ is an external force (periodic excitation of pulsation $\omega)$. $\mathbf{q}(t)$ defines the breathing behavior of the global stiffness $\mathbf{K}_{c}$ at the crack location due to the evolution of the external forces. If we assume the open crack at the $i^{\text {th }}$ element, the global stiffness matrix $\mathbf{K}_{c}$ defines the global stiffness matrix due to the presence of the crack in the fixed frame $(X, Y, Z)$. It is given by

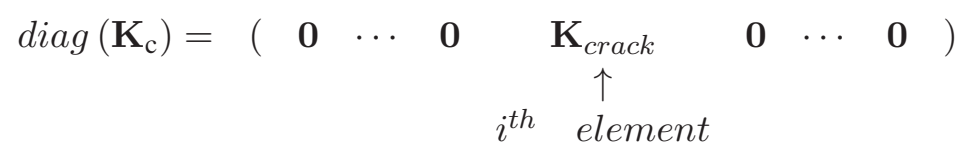

In the present study, it is assumed that the opening and closing of the crack depends on the level of load $[14,15]$. The crack breathing phenomenon varies with time due to vibration. Examining the dynamic response of a breathing crack at its first mode, it can be decomposed in the following way [15]

$$
\mathbf{q}(t)=\frac{1}{2}\left(\mathbf{K}_{c}-\mathbf{K}\right)(1-\cos \omega t)
$$

where $\omega$ is equal to the excitation and is defined as the crack breathing frequency. If $(1-\cos \omega t)=0$ (i.e. $\omega t=2 n \pi$ with $n$ any integer), the crack is closed and has no effect on the dynamic behaviour: the beam may be treated as uncracked. If $(1-\cos \omega t)=1$ (i.e. $\omega t=(2 n-1) \pi$ with $n$ any integer), the crack is in the fully open state. Otherwise the crack is in a state of partial closure given by the relation (3).

The modeling of a transverse crack that was described extensively in $[16,17]$ is briefly presented here. The model proposed by Mayes and Davies $[18,19]$ is used in order to locally represent the stiffness properties of the crack cross section. Due to strain energy concentration in the vicinity of the tip of the crack under load, the presence of a transverse crack introduces local flexibility. So, this model considers the reduction of the second moment of area $\Delta I$ of the element at the location of the crack that may be defined by

$$
\Delta I=I_{0}\left(\frac{\frac{R}{l}\left(1-\nu^{2}\right) F(\mu)}{1+\frac{R}{l}\left(1-\nu^{2}\right) F(\mu)}\right)
$$

where $I_{0}, R, l$, and $\nu$ are the second moments of area, beam external radius, length of the section and Poisson's ratio, respectively. $\mu$ is the non-dimensional crack depth and is given by $\mu=\frac{h}{R}$ where $h$ defines the crack depth of the beam, as shown in Figure 1(b). As indicated in Figure 1(b), the total angle $\alpha$ subtended by the crack is related to the depth of crack by the relation $\alpha=2 \cos ^{-1}(1-\mu) . F(\mu)$ defines the non-linear compliance as a function of variations in non-dimensional crack depth $\mu$, which can be derived from a series of experiments using chordal cracks (see Mayes and Davies $[18,19]$ ). Considering the finite element formulation and the local reduction of the second moments of area that are given in Equation (4), the stiffness contribution $\mathbf{K}_{c r a c k}^{\eta, \xi}$ of an open crack about the centroidal axes $\eta$ and $\xi$ for a two nodes Timoshenko beam element of length $l$ and Young's modulus $E$ (corresponding to the flexible degree-of-freedom $\left[u_{1} ; v_{1} ; \theta_{1} ; \psi_{1} ; u_{2} ; v_{2} ; \theta_{2} ; \psi_{2}\right]$, see Figure 1(c)) can be defined at the crack location

$$
\mathbf{K}_{\text {crack }}^{\eta, \xi}=\frac{E}{l^{3}}\left(\begin{array}{cccccccc}
12 I_{\eta} & 0 & 0 & 6 l I_{\eta} & -12 I_{\eta} & 0 & 0 & 6 l I_{\eta} \\
& 12 I_{\xi} & -6 l I_{\xi} & 0 & 0 & -12 I_{\xi} & -6 l I_{\xi} & 0 \\
& & 4 l^{2} I_{\xi} & 0 & 0 & 6 l I_{\xi} & 2 l^{2} I_{\xi} & 0 \\
& & & 4 l^{2} I_{\eta} & -6 l I_{\eta} & 0 & 0 & 2 l^{2} I_{\eta} \\
& & & & 12 l I_{\eta} & 0 & 0 & -6 l I_{\eta} \\
& & & & & 12 l I_{\xi} & 6 l I_{\xi} & 0 \\
& & & & & & 4 l^{2} I_{\xi} & 0 \\
& & & & & & & 4 l^{2} I_{\eta}
\end{array}\right)
$$


Finally, $\mathbf{K}_{\text {crack }}$ that is defined in Equation (2) is obtained by passing from the fixed frames $(\eta, \xi, Z)$ to $(X, Y, Z)$ via the rotation $\chi$ (see Figure 1(b) for more details).

\begin{tabular}{ccc}
\hline Notation & Description & Value \\
\hline$R_{e}$ & outer radius of the beam & $0.1 \mathrm{~m}$ \\
$R_{i}$ & inner radius of the beam & $0.06 \mathrm{~m}$ \\
$L$ & length of the rotor shaft & $0.5 \mathrm{~m}$ \\
$E$ & Young's modulus of elasticity & $2.110^{11} \mathrm{~N} . \mathrm{m}^{-2}$ \\
$\rho$ & density & $7800 \mathrm{~kg} \cdot \mathrm{m}^{-3}$ \\
$\nu$ & Poisson ratio & 0.3 \\
$\zeta$ & modal damping & $10^{-3}$ \\
\hline
\end{tabular}

Table 1: Value of the physical parameters

\section{Determination of the nonlinear response of the cracked beam}

As previously indicated, the above equations of the cracked pipeline beam have a time-dependent coefficient due to the fact that the crack breathes when the excitation force changes. The non-linear dynamic response of the system can be approximated by finite Fourier series

$$
\mathbf{x}(t)=\sum_{k=1}^{m}\left(\mathbf{A}_{k} \cos (k \omega t)+\mathbf{B}_{k} \sin (k \omega t)\right)
$$

where $\omega$ defines the crack breathing frequency. $\mathbf{A}_{k}$ and $\mathbf{B}_{k}$ (with $k=1, \cdots, m$ ) define the unknown coefficients of the finite Fourier series. The number of harmonic coefficients $m$ is selected on the basis of the number of significant harmonics expected in the dynamical response of the cracked beam. The excitation force f can be exactly defined by finite Fourier series with first-order periodic components in the frequency domain. Expressions are given by $\mathbf{f}=\mathbf{C}_{1} \cos (\omega t)+\mathbf{S}_{1} \sin (\omega t)$ with $\mathbf{C}_{1}$ and $\mathbf{S}_{1}$ the first-order periodic components in the frequency domain.

As previously explained, the amount of open part of the crack constantly varies with the excitation force, thereby changing the stiffness $\mathbf{q}(t)$ of the cracked beam. So, by considering the approximated nonlinear solution of the cracked beam (see Equation (6)) and the global mechanism of the time dependent part for the crack breathing phenomenon (see Equation (3)), the expression $\mathbf{q}(t) \mathbf{x}(t)$ can be approximated by the following expression

$$
\mathbf{q}(t) \mathbf{x}(t)=\frac{1}{2}\left(\mathbf{K}_{c}-\mathbf{K}\right)(1-\cos \omega t)\left(\sum_{k=1}^{m}\left(\mathbf{A}_{k} \cos (k \omega t)+\mathbf{B}_{k} \sin (k \omega t)\right)\right)
$$

By substituting expressions (6) and (7) in Equation (1), the unknown coefficients of the finite Fourier series $\mathbf{A}_{k}$ and $\mathbf{B}_{k}$ (with $k=1, \cdots, m$ ) can be found solving the following equations

$$
\begin{aligned}
& \left(\mathbf{K}-k^{2} \omega^{2} \mathbf{M}\right) \mathbf{A}_{k}-k \omega \mathbf{C B}_{k}+\left(\mathbf{K}_{c}-\mathbf{K}\right)\left(\frac{\mathbf{A}_{k-1}}{4}+\frac{\mathbf{A}_{k}}{2}+\frac{\mathbf{A}_{k+1}}{4}\right)=\delta_{k=1} \mathbf{C}_{1} \\
& k \omega \mathbf{C} \mathbf{A}_{k}+\left(\mathbf{K}-k^{2} \omega^{2} \mathbf{M}\right) \mathbf{B}_{k}+\left(\mathbf{K}_{c}-\mathbf{K}\right)\left(\frac{\mathbf{B}_{k-1}}{4}+\frac{\mathbf{B}_{k}}{2}+\frac{\mathbf{B}_{k+1}}{4}\right)=\delta_{k=1} \mathbf{S}_{1}
\end{aligned}
$$

with $\mathbf{A}_{n}=\mathbf{0}$ and $\mathbf{B}_{n}=\mathbf{0}$ if $n<1$. The complete algorithm and process of the computation is given in Figure 2. 


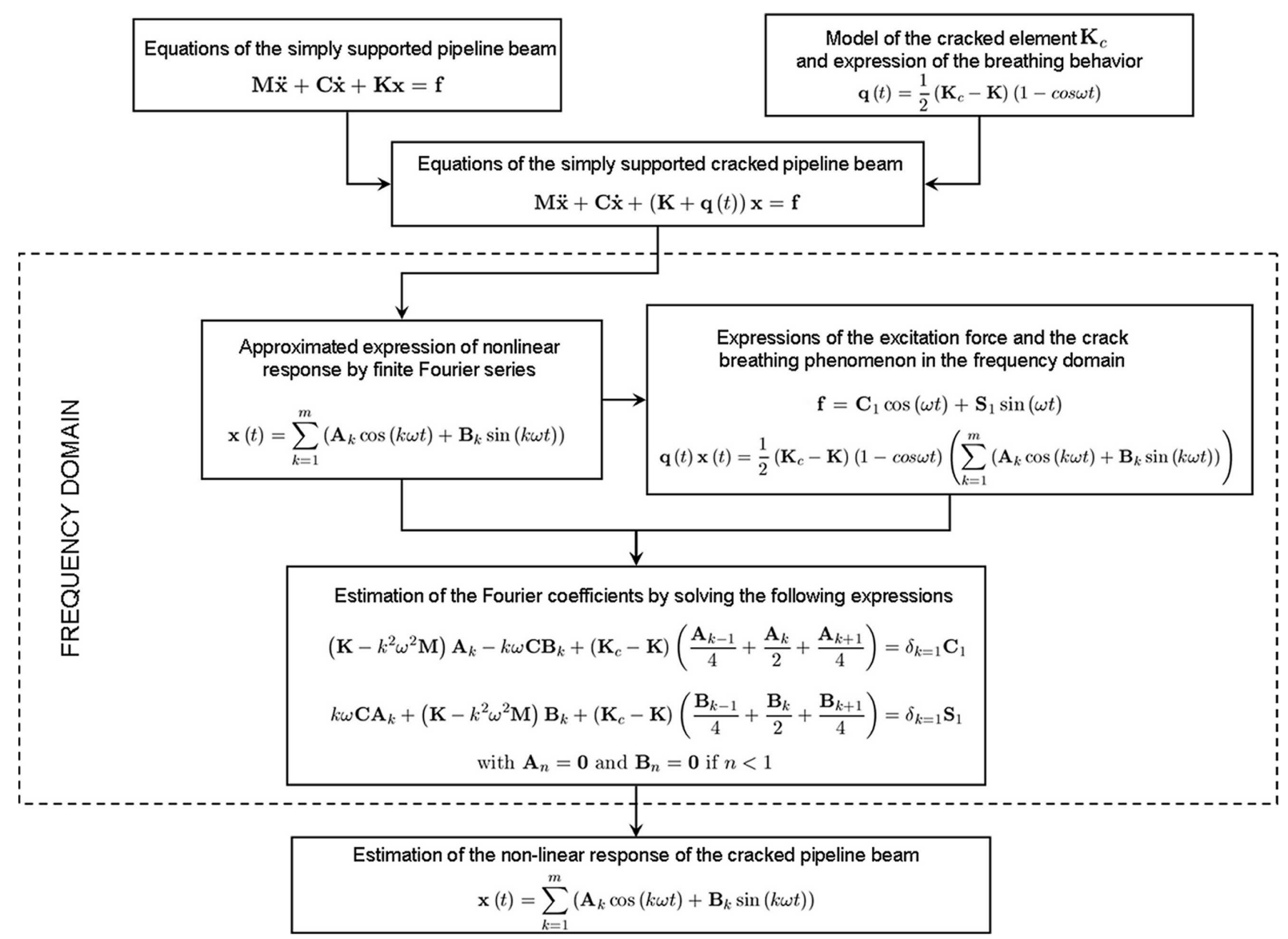

Figure 2: Process of the numerical computation for the calculation of the non-linear displacements of the simply supported cracked pipeline beam

\begin{tabular}{ccccccc}
\hline Case & Crack element & $L_{\text {crack }}(\mathrm{m})$ & $\mu$ & $\chi$ & Excitation element & $L_{\text {excitation }}(\mathrm{m})$ \\
\hline 1 & 7 & 0.1625 & 0.3 & 0 & 10 & 0.25 \\
2 & 10 & 0.2375 & 0.1 & 0 & 10 & 0.25 \\
3 & 7 & 0.1625 & 0.3 & $\frac{\pi}{3}$ & 10 & 0.25 \\
4 & 15 & 0.3625 & 0.3 & $\frac{\pi}{3}$ & 2 & 0.05 \\
\hline
\end{tabular}

Table 2: Description of cases

\section{Numerical results}

In this section, the effects of a breathing crack on the nonlinear dynamic response of the beam are quantified. The basic deterministic parameters used in all cases are given in Table 1. For each case, the location of the external force, the position, orientation and depth of the crack are given in Table 2. First of all, the detection of the presence of one crack by using the appearance of the non-linear components will be investigated. Secondly, we undertake the determination of the crack location based on the appearances of anti-resonances in the signature of the non-linear components. 


\subsection{Crack detection based on the non-linear response}

First of all, we consider a horizontal crack $\left(\chi=0\right.$ degree) with a crack severity $\mu=0.3$ at the $7^{\text {th }}$ element (case 1 given in Table 2). Figure 3(a) shows the non-linear response for the pipeline beam with the breathing crack. It clearly appears that the global response is composed by not only the first harmonic component but also by the components of higher order. For example, the second, third and fourth orders are predicted when the crack breathing frequency is approximately one-half, one-third and one-fourth of the first resonance frequency, as indicated in Figure 3(a). Moreover, due to the presence of the crack, the $n^{\text {th }}$ orders increase when the frequency of the excitation force is near $\frac{1}{m}$ of the first resonance frequency (with $m<n$ or any $m$ positive integer). It is recalled that the response will be composed by only the first harmonic component (i.e. a linear response) for the uncracked beam. Then, Figure 3(b) shows the non-linear response for a small crack that is situated at the $10^{\text {th }}$ element with $\chi=0$ degree (see case 2 in Table 2). It is observed that the global nonlinear response has a significant peak at one-half of the first frequency of the cracked system. However, only small changes in the vibration amplitude are observed at one-third or one-fourth of the first frequency. So, it may be concluded that the non-linear behavior for a small crack is mainly due to the predominance of the first and second harmonic components. Therefore it should be considered that contributions of the third and fourth harmonic components are too small to allow the detection of crack in realistic cases.

Now, we consider case 3 given in Table 2. Compared to the previous case 1, the crack orientation $\chi$ that defines the angle between the orientation of the crack front and the vertical axis (see Figure 1(b)) has changed. Figures 4(a) and 4(b) show some interesting features for the coupling measurements (i.e. horizontal and vertical directions) of the nonlinear components due to the presence of the crack. Indeed, the presence of the crack introduces excitation on the principal $\mathrm{X}$-axis (i.e. horizontal displacement) even if the external force is only on the principal Y-axis (i.e. vertical displacement). The appearance of nonlinear vibrations in the horizontal direction is only due to the breathing behavior of the crack and the fact that the crack front is not in the same direction than the external force (i.e. $\chi \neq 0$ ). In the two previous cases (i.e. cases 1 and 2 ), there is no coupling between the two lateral vibrations due to the fact that the external force is on the same direction than the crack front (i.e. $\chi=0$ ). Thereby, the crack does not induce excitation on the principal horizontal axis. So no vibration on the $\mathrm{X}$-axis exists.

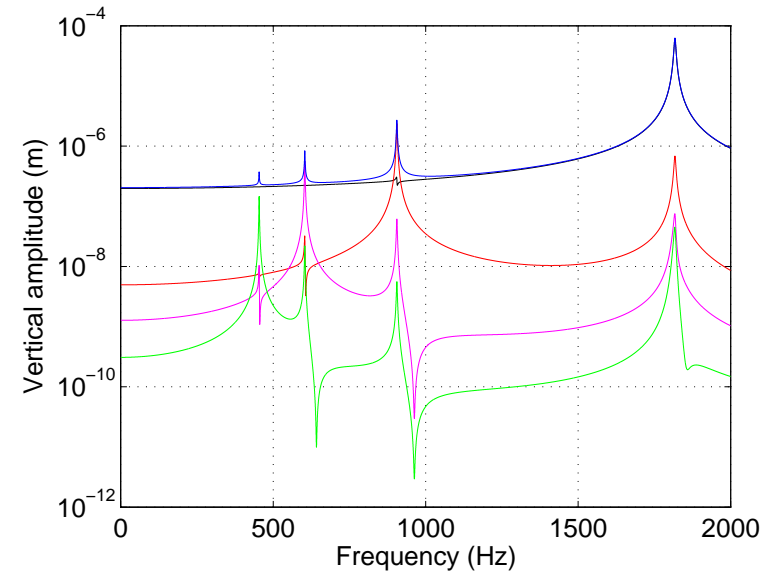

(a) Case 1

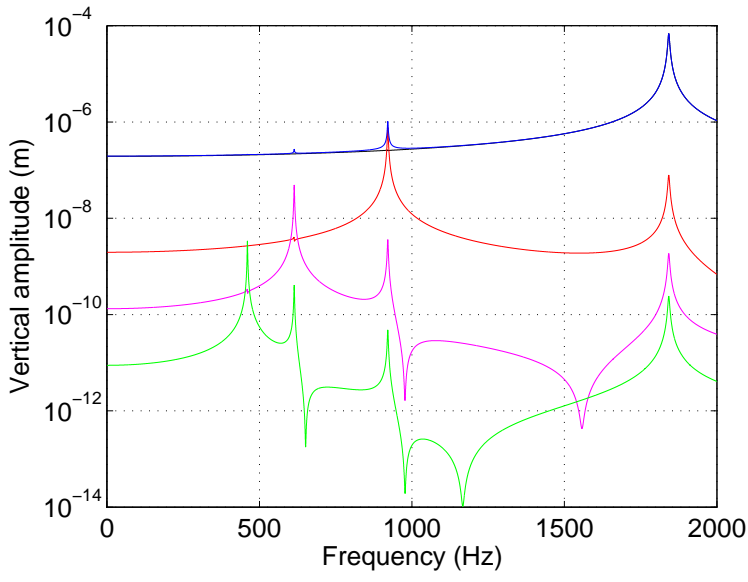

(b) Case 2

Figure 3: Non-linear vertical amplitudes for cases 1 and 2 at the middle of the beam (blue=global non-linear response, black $=$ order 1 , red $=$ order 2 , magenta $=$ order 3 , gre en $=$ order 4 ) 


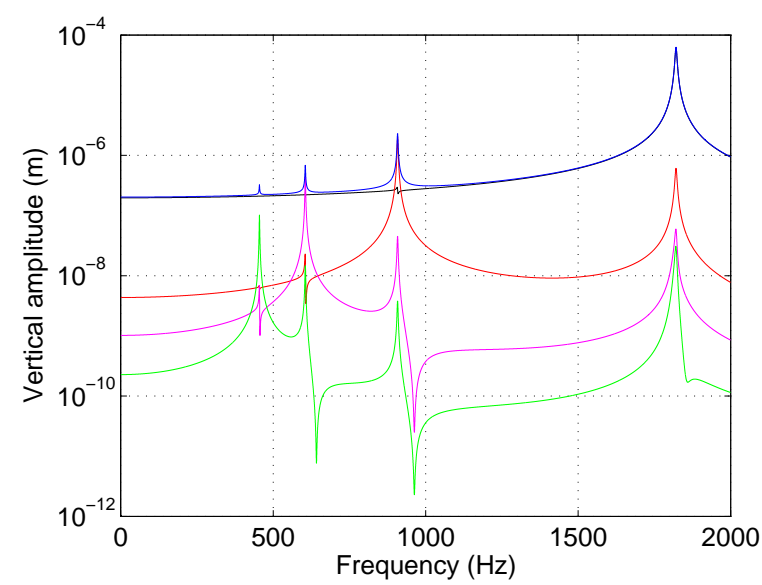

(a) Vertical displacement

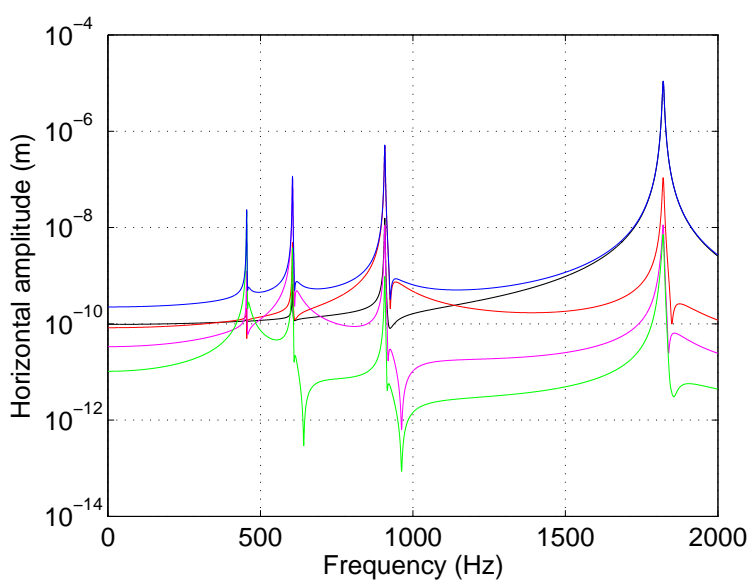

(b) Horizontal displacement

Figure 4: Non-linear vertical and horizontal amplitudes for case 3 at the middle of the beam (blue=global non-linear response, black=order 1 , red=order 2 , magenta $=$ order 3 , green=order 4 )

\subsection{Crack location based on the anti-resonances of the non-linear components}

In this part of the paper, we propose to use evolutions of anti-resonances of the Higher-order Frequency Response Functions for detecting the damage location.

Indeed, it is well known that the breathing behavior of the crack induces an additional internal force for the linear and non-linear components at the crack location [20]. Due to the fact that the external force $f$ given in Equation (1) only induces first-order periodic components in the frequency domain, it can be concluded that only the presence of the breathing crack adds an excitation for higher orders. So, the position of the crack can be identified by considering the evolution of anti-resonances of the super-harmonic components.

To illustrate this, Figures 5(a), 5(b) and 5(c) give the evolutions of the second, third and fourth harmonic components of the cracked beam in the vertical axis for case 2. First of all, the appearances of peak amplitudes for the second, third and fourth harmonic components that form the signature of the presence of a crack are visible for all the positions of the pipeline beam. Secondly, it appears that the emerging and location of some new anti-resonances and the variation in the anti-resonance frequency along the beam depend on the crack location. For example, it can be observed that the anti-resonance frequency decrease from $3300 \mathrm{~Hz}$ to $2200 \mathrm{~Hz}$ for the variations of the beam location from $0 \mathrm{~m}$ to $0.25 \mathrm{~m}$. Then increased values of anti-resonance frequency is observed along the beam (from $0.25 \mathrm{~m}$ to $0.5 \mathrm{~m}$ ). The minimum of anti-resonances (in frequency) for the second order harmonic components of the cracked system appears at the crack location (see the red circle in Figure 5(a)). The same conclusions can be made for the third and fourth harmonic components, as indicated in Figures 5(b) and 5(c). Here again, the red circle indicated the minimum of anti-resonance frequency for the third and fourth-order Frequency Response Functions. So it can be concluded that the beam position where the minimum value of anti-resonance frequency is observed for all the non-linear harmonic components corresponds to the location of the crack.

Then, Figures 6(a), 6(b), 6(c), 6(d), 6(e) and 6(f) give the evolutions of all harmonic components in the vertical and horizontal directions for case 3 . It is recalled that the presence of horizontal responses of the system is due to the coupling measurements via excitation of the crack in the horizontal axis and the breathing behavior. In both directions and for all harmonic components, the minimum value of anti-resonance frequency is detected at the position of the crack (see the red circle for all Figures 6).

Now, we consider case 4 that is concerns with a different position for both the crack location and the external excitation. The same behavior for the variation of the anti-resonance frequency is observed in case 4, 


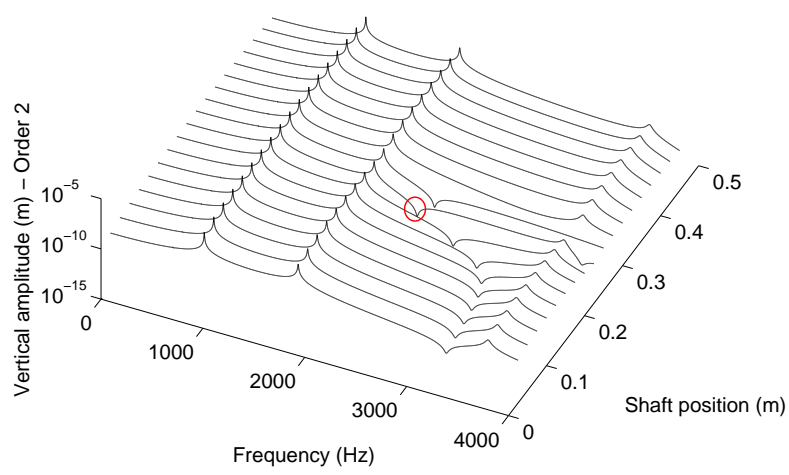

(a) Order 2

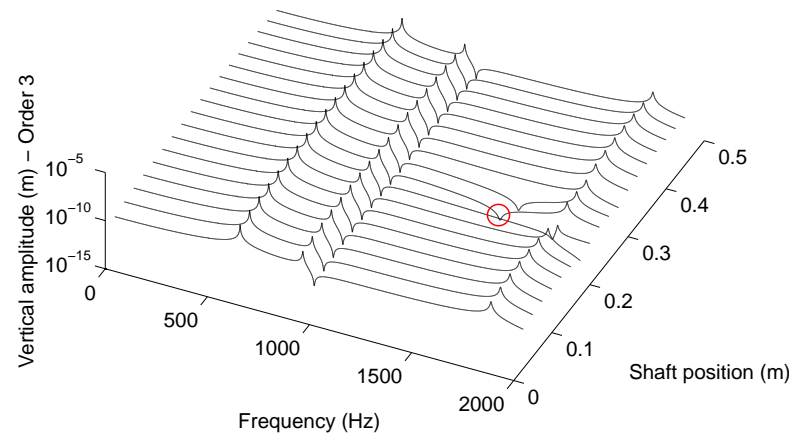

(b) Order 3

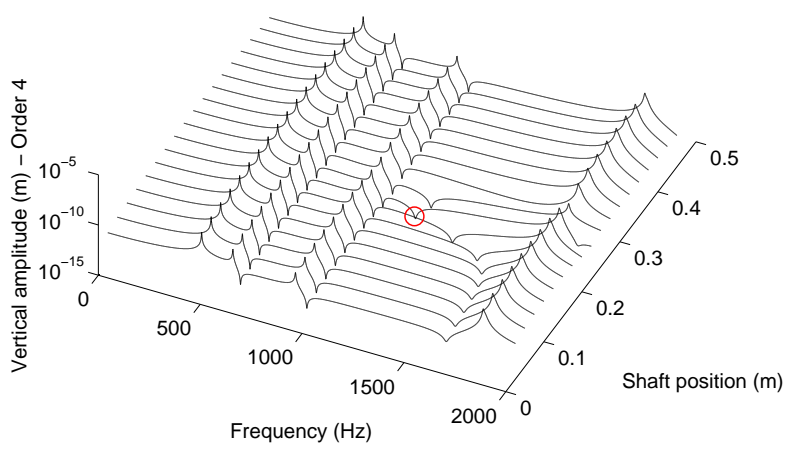

(c) Order 4

Figure 5: Vertical displacement for all the beam positions - Case 2

as illustrated in Figures 7(a), 7(b), 7(c), 7(d), 7(e) and 7(f). The minimum of the anti-resonance frequency is located at the crack position indicated in the previous figures by the red circle.

Considering all these results, it can be concluded that one of the main advantage of using the anti-resonances for the Higher-order Frequency Response Functions is that the detection of the crack location is not sensitive to the excitation position and can be easily applied for the detection of small cracks.

Finally, Figure 8 gives the first harmonic component in the horizontal direction for case 4 . Due to the fact that the external excitation is in the vertical direction, the appearance of the first harmonic component in the horizontal direction is only due to the crack. So, the position of the minimum of anti-resonance frequency gives the crack location (indicated by the red circle in Figure8). For all the present study, it is also remained that the breathing behavior given in Equation (3) is only valuable around the first frequency. So all the previous comments discussed in the present section are limited to the non-linear dynamic behavior of the cracked system around its first frequency.

\section{Conclusion}

This paper highlights the possibility of crack detection through the observation of the non-linear dynamic behaviour of a simply supported cracked pipeline beam. This study has demonstrated that the introduction of 


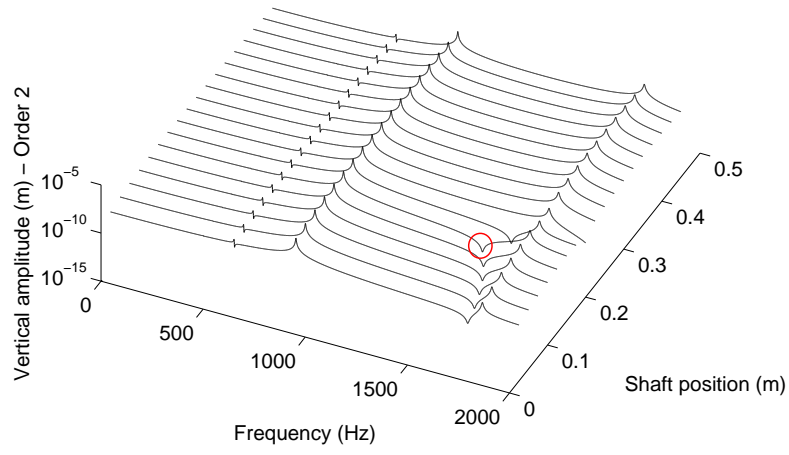

(a) Vertical displacement - Order 2

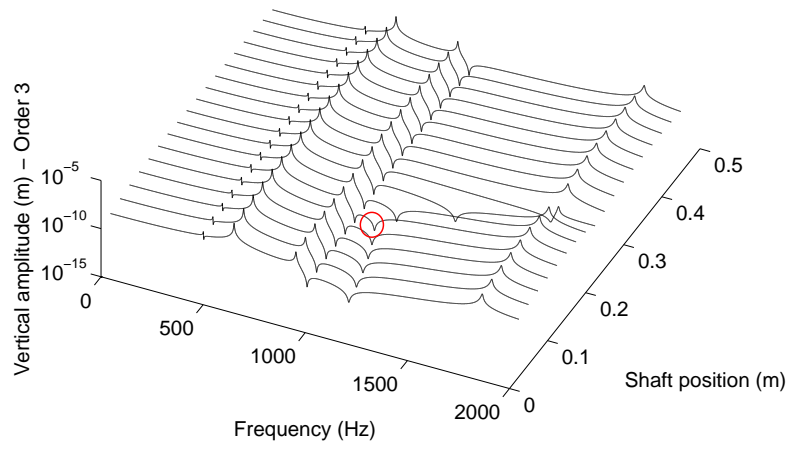

(c) Vertical displacement - Order 3

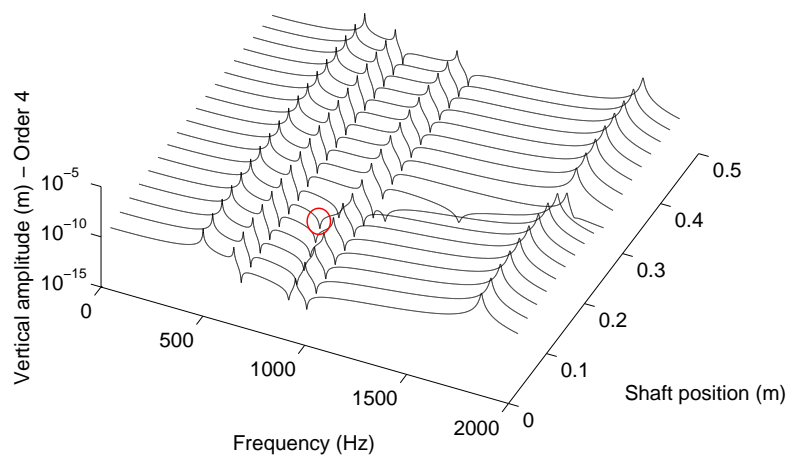

(e) Vertical displacement - Order 4

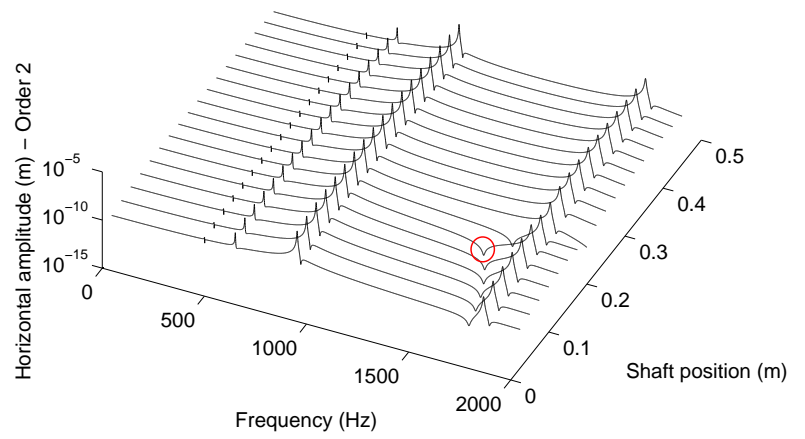

(b) Horizontal displacement - Order 2

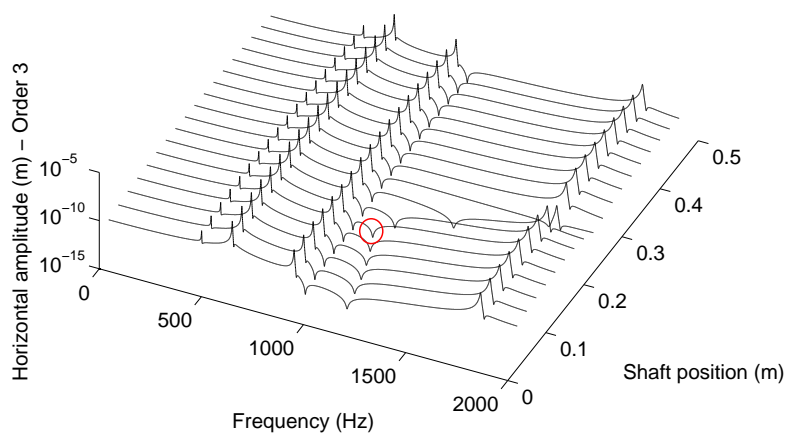

(d) Horizontal displacement - Order 3

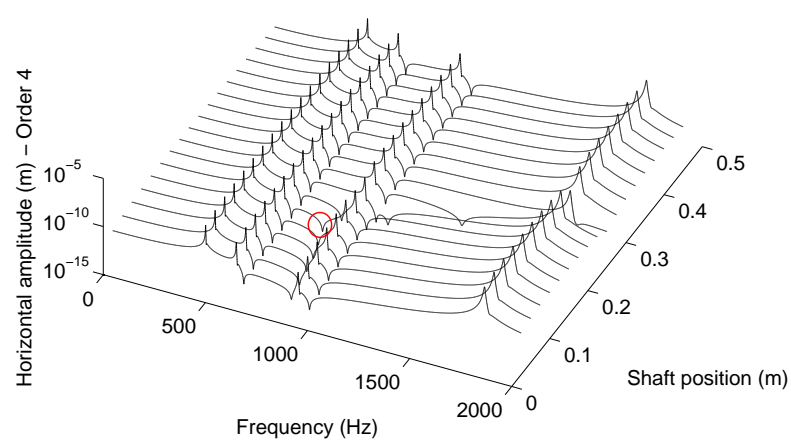

(f) Horizontal displacement - Order 4

Figure 6: Vertical and horizontal displacements for all the beam positions - Case 3 


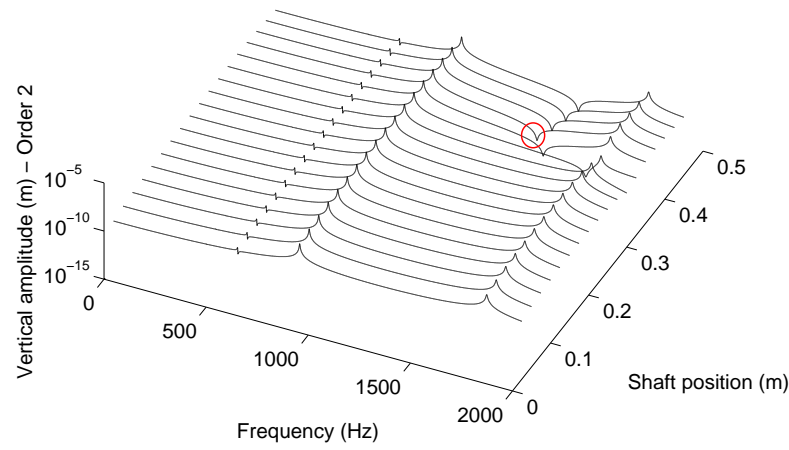

(a) Vertical displacement - Order 2

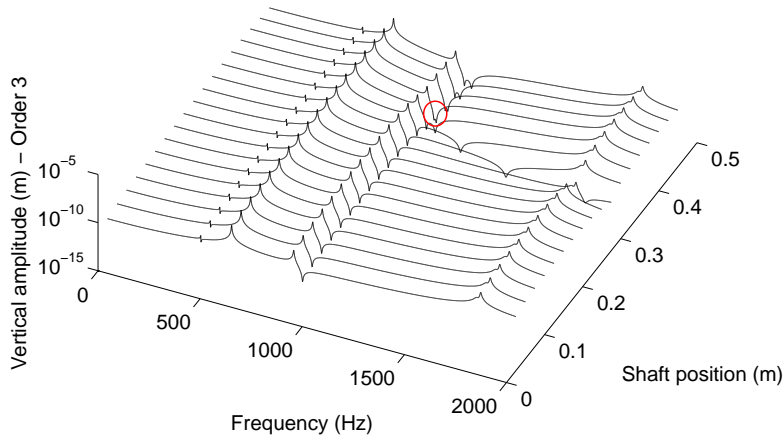

(c) Vertical displacement - Order 3

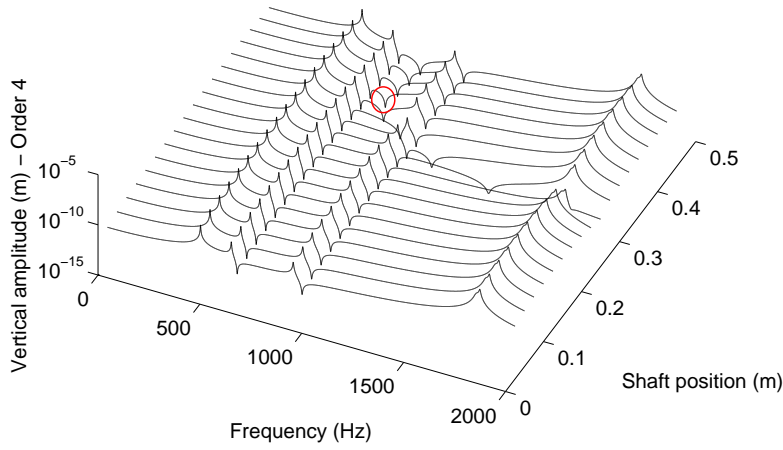

(e) Vertical displacement - Order 4

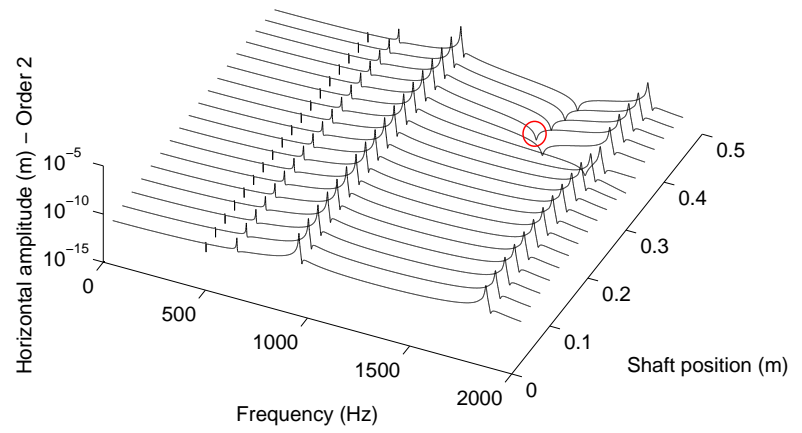

(b) Horizontal displacement - Order 2

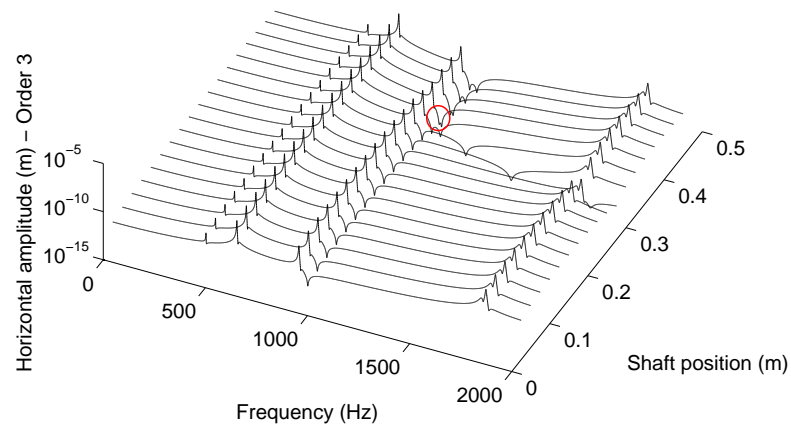

(d) Horizontal displacement - Order 3

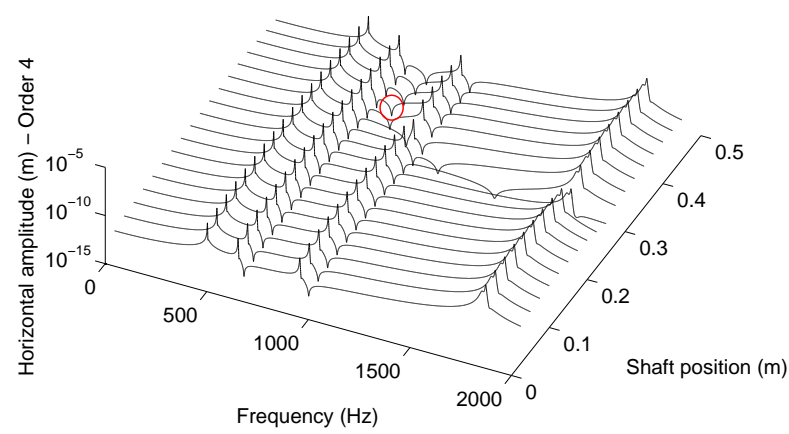

(f) Horizontal displacement - Order 4

Figure 7: Vertical and horizontal displacements for all the beam positions - Case 4 


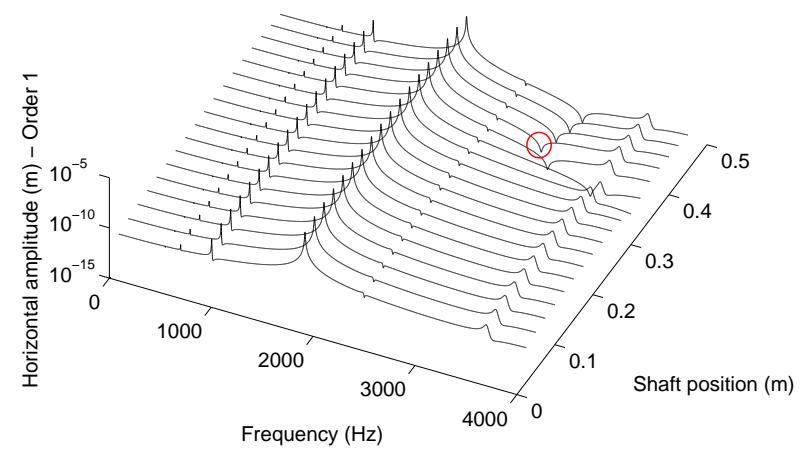

Figure 8: Vertical displacement of the first harmonic component - Case 4

a crack breathing phenomenon that varies with time due to vibration induces the emerging of the $n^{\text {th }}$ superharmonic frequency components. The appearances of non-linear components can be considered as one of the most practical indicators of the presence of a crack for health monitoring purposes.

The localization of anti-resonances for Higher-Order Frequency Response Functionsn provides useful information on the location of a crack and can be used on an on-line robust crack monitoring system.

\section{References}

[1] Doebling, S., Farrar, C., Prime, M., and Shevits, D., 1996. "Damage identification and health monitoring of structural and mechanical systems from changes in their vibration characteristics: A literature review". Los Alamos National Laboratory, USA, 1, pp. 1-136.

[2] Doebling, S., Farrar, C., Prime, M., 1998. "A summary review of vibration-based damage identification methods". Shock and Vibration Digest, 30, p. 91-105.

[3] Dimarogonas, A., 1996. "Vibration of cracked structures: a state of the art review". Engineering Fracture Mechanics, 55, p. 831-857.

[4] Sinou J.-J., 2009. A review of damage detection and health monitoring of mechanical systems from changes in the measurement of linear and non-linear vibrations. In: Mechanical Vibrations: Measurement, Effects and Control, Chapter 13. Nova Science Publishers, Editor: Robert R. Sapri, pp 643-702.

[5] Salawu, O., 1997. "Detection of structural damage through changes in frequency: a review". Engineering Structures, 19(9), p. 718-723.

[6] Gounaris, G. D., Papadopoulos, C. A., and Dimarogonas, A. D., 1996. "Crack identification in beams by coupled response measurements". Computers and Structures, 58(2), p. 299-305.

[7] Liu, D., Gurgenci, H., and Veidt, M., 2003. "Crack detection in hollow section structures through coupled response measurements". Journal of Sound and Vibration, 261, p. 17-29.

[8] Bamnios, Y., Douka, E., and Trochidisi, A., 2002. "Crack identification in beam structures using mechanical impedance”. Journal of Sound and Vibration, 256 (2), p. 287-297. 
[9] Dharmaraju, N., and Sinha, J. K., 2005. "Some comments on use of anti-resonance for crack identification in beams". Journal of Sound and Vibration, 286, p. 669-671.

[10] Dilena, M., and Morassi, A., 2004. "The use of anti-resonances for crack detection in beams". Journal of Sound and Vibration, 276, p. 195-214.

[11] Gudmundson, P., 1983. "The dynamic behaviour of slender structures with cross sectional cracks". Journal of Mechanics and Physics of Solids, 31, p. 329-345.

[12] Pugno, N., Surace, C., and Ruotolo, R., 2000. "Evaluation of the non-linear dynamic response to harmonic excitation of a beam with several breathing cracks". Journal of Sound and Vibration, 235(5), p. 749-762.

[13] Sinha, J., and Friswell, M., 2002. "Simulation of the dynamic response of a cracked beam". Computers and Structures, 80, p. 1473-1476.

[14] Elber, W., 1970. "Fatigue crack closure under cyclic tension.". Engineering Fracture Mechanics, 2, pp. $37-45$.

[15] Cheng, S.M., Wu, X.J. and Wallace, W., 1999. "Dynamics of cracked rotors: Literature survey". Journal of Sound and Vibration, 225(1), pp. 201-208.

[16] Sinou, J.-J., 2007. "Numerical investigations of a robust identification of crack location and size in beams using only changes in ratio pulsations of the cracked beams". Structural Engineering Mechanics, 25(6), pp. 691-716.

[17] Sinou, J.-J., 2007. "Damage Assessment Based on the Frequencies' Ratio Surfaces Intersection Method for the Identification of the Crack Depth, Location and Orientation". Structural Durability and Health Monitoring, 3(3), pp. 134-162.

[18] Davies, W. G. R., and Mayes, I. W., 1984. "The vibrational behaviour of a multi-shaft, multi-bearing system in the presence of a propagating transverse crack". Transactions of the ASME Journal of Vibration, Acoustics, Stress, and Reliability in Design, 106, pp. 146-153.

[19] Mayes, I. W., and Davies, W. G. R., 1984. "Analysis of the response of a multi-rotor-bearing system containing a transverse crack in a rotor". Transactions of the ASME Journal of Vibration, Acoustics, Stress, and Reliability in Design, 106, pp. 139-145.

[20] Sinou, J.-J., 2008. "Detection of cracks in rotor based on the $2 \mathrm{X}$ and $3 \mathrm{X}$ super-harmonic frequency components and the crack-unbalance interactions". Communications in Nonlinear Science and Numerical Simulation, 13, pp 2024-2040 